\title{
A lux/gfp dual label system for studying attachment and biofilm formation of Enterobacter sakazakii
}

\author{
Melanie Thimodo
}

\author{
This manuscript was prepared under the supervision of Prof. Mansel Griffiths and \\ Dr. Haifeng Wang, Canadian Research Institute for Food Safety, Ontario Agricultural College.
}

\begin{abstract}
A significant feature of Enterobacter sakazakii is the ability to form biofilms enabling its persistence in manufacturing and neonatal environments, and implicating it as the primary cause of outbreaks of neonatal meningitis. Conventional methods of studying E. sakazakii are time consuming, inaccurate and damaging to cells, preventing further analysis. A novel method for detecting biofilm formation has been utilized that takes advantage of a dual lux/gfp reporter system cloned into cells for simultaneous quantification of bacterial metabolic activity and cell numbers respectively. To evaluate the effectiveness and accuracy of the novel method compared to the conventional method, strains of bacteria were allowed to form biofilms and were measured using both methods. Biofilm formation of E. sakazakii strains over 2 days was measured and peak formation and changes in biofilm development was determined for each strain. The lux reporter was utilized to determine metabolic activity of cells in the biofilm, correlating to biofilm formation, over 3 days. Biofilm formation of strains was measured using both methods and compared to detect trends. This study found that the novel method was effective at detecting changes in metabolic activity of cells in biofilm. Cell numbers were to be simultaneously detected via the $g f p$ reporter but filters with correct detection wavelength were unavailable thus cell numbers were not obtained here. These observations determine that this dual reporter system is a promising tool for monitoring bacteria in situ and for further understanding of biofilm formation.
\end{abstract}

$E$ nterobacter sakazakii is an opportunistic organism found in food processing environments associated with cases of necrotizing enterocolitis, bacteraemia and meningitis in infants. ${ }^{3,5,7}$ E. sakazakii has been detected in various types of foods and although little is known about modes of transmission, dried-infant formula milk (IFM) is the only food that has been linked in incidents of transmitted disease causing neonatal meningitis. ${ }^{5,7,9}$ Even with low reports of $E$. sakazakii in most countries due to lack of surveillance systems, between 1961 and 2003 there were 48 reported cases of this illness. With a low frequency of 1 per 100,000 infants contracting the disease, the results are still devastating with a reported mortality rate between 20 to $>50 \%,{ }^{5,9}$ with some as high as $80 \%$. Survivors suffer severe neurological sequelae such as hydrocephalus, quadriplegia and retarded neural development. ${ }^{2,5}$ Infants at high risk for contracting meningitis from E. sakazakii are usually less than one year old and are particularly susceptible during the first 28 days. Low birth weight, premature and immunocompromised infants as well as infants with HIV-positive mothers are at the highest risk. ${ }^{9}$ A study in 120 samples of infant formula in Canada found $6.7 \%$ to be positive with $E$. sakazakii. $^{7}$ Outbreaks of necrotizing enterocolitis and meningitis caused by E. sakazakii have also occurred in neonatal intensive care units due to the persistence and biofilm formation in IFM and feed preparation equipment such as brushes, blenders and spoons. ${ }^{2}$ The effects of the disease and the persistence of the bacteria in both the manufacturing process and in medical facilities due to biofilm formation make it important to develop methods to study and understand biofilms.

Biofilms are a constant concern in food processing environments, acting as a possible source of contamination that may lead to food spoilage or the transmission of disease. ${ }^{4}$ Biofilm formation on surfaces has been suggested as a survival strategy for several food associated organisms like Listeria monocytogenes and Escherichia coli. ${ }^{1,4,5}$ Biofilms are formed after the attachment of bacteria to equipment and their survival is dependent on the interactions between the bacterial cell, the surface and the surrounding microenvironment. ${ }^{3,4,6}$ E. sakazakii secretes a viscous, extrapolysaccharide, capsular material that enhances the attachment and adherence to surfaces, ${ }^{2}$ and provides a physical barrier protecting cells from environmental stresses placed upon them in food processing environments like UV light, osmotic stress, heat, detergents and antibiotics. ${ }^{3,5,6}$ Biofilms formed by E. sakazakii provide them with the ability to adapt to high osmotic potentials, dry conditions, air 
drying and other desiccation conditions compared with other members of Enterobaceriaceae. ${ }^{3,5,9}$ E. sakazakii is also more thermotolerant than other Enterobacteriaceae attributed by its survival after heat treatments, and its lowest growth temperature at $5.5^{\circ} \mathrm{C}$, increasing it potential to grow when refrigerated. $^{2}$ Biofilms protect $E$. sakazakii from manufacturing processes intended to kill them, and when rehydrated for consumption they retain pathogenicity and cause infection. When grown in IFM, E. sakazakii was found to adhere strongly to silicon, latex, polycarbonate and stainless steel, materials commonly used for infant feeding equipment and IFM preparation areas. ${ }^{2,9}$ The efficacies of sanitizers are also reduced by the presence of biofilms, making it difficult to eliminate bacterial contamination in food processing environments. ${ }^{4}$

Conventional methods for detecting biofilm thus far involve growing the bacteria at different time intervals, multiple washing and drying stages, the addition of a dye like crystal violet solution, destaining and measuring optical density where absorbance values correlate to biofilm formation. ${ }^{1,2}$ These processes are very time consuming, and the dyes used are very strong staining reagents that can give false positives or inaccurate results. Performing analyses at different time intervals is also difficult because once stained and measured, bacteria are no longer viable. Such obstacles make it challenging to study growth patterns and conditions that affect biofilm formation by $E$. sakazakii. The development of a novel, effective and rapid system to detect and study E. sakazakii biofilm formation is essential and crucial in containing their persistence and subsequently the spread of disease.

A novel method for monitoring the formation and growth limits of E. sakazakii has been proposed by using a dual fluorescent marker system as described by Unge et al. ${ }^{10}$ This system marks cells with lux and $g f p$ genes, encoding for bacterial luciferase and green fluorescent protein (GFP) respectively. ${ }^{10}$ The advantages of this marking system are two-fold; the lux gene codes for bioluminescent bacterial luciferase which is dependent on cellular energy and proportional to biomass, thus the bioluminescence output can be directly correlated to the metabolic activity of the cells. ${ }^{10}$ The $g f p$ gene produces a fluorescent protein that does not require the addition of a substrate or cofactor in order to fluoresce and has no energy requirement, thus it will fluoresce under all conditions including the stationary phase or during starvation. ${ }^{10}$ This new system takes advantage of the properties of the two markers and will simultaneously be able to measure metabolic activity and cell numbers to better understand biofilm formation, so that improved processes can be implemented to deter growth and prevent contamination.

It is important to understand the interactions between bacteria and surfaces and their impacts on the effectiveness on cleaning and sanitizing to provide more effective measures for biofilm prevention and removal. ${ }^{4}$ Biofilm formation in food processing environments is of special importance because these biofilms can act as chronic sources of microbial contamination. In this study a total of 8 strains of wild type E. sakazakii were utilized and optimized using the old methods to be used as a comparison system to the proposed new system. The new system uses 4 strains of $E$. sakazakii cloned with the dual marker genes to determine its potential and effectiveness for rapidly detecting and studying E. sakazakii biofilm formation. This new method will be used to investigate the effects of $\mathrm{pH}$, temperature, desiccation, osmotic pressure and nutrients on biofilm formation and can be utilized to develop new environments in the manufacturing process.

\section{MATERIALS AND MeTHODS}

\section{Bacterial strains and culture conditions}

\section{E. sakazakii bacterial strains}

A total of 8 strains of E. sakazakii were obtained, 7 were kindly provided by Dr. Franco J. Pagotto, Health Canada, Ottawa, coded ES 2855, ES 2870, ES 2871, ES 2876, ES 3253, ES 3263 and ES 5199 and 1 strain, ES 51329 was purchased from American Type Culture Collection (ATCC).

\section{Bioluminescent E. sakazakii strains}

A total of 4 strains of $E$. sakazakii were transformed with a plasmid, pGluxAK harboring the luxCDABE and $g f p$ genes at the Canadian Research Institute for Food Safety (CRIFS), University of Guelph as preliminary steps for this project. Strains were 2876-3 AO 8-12, 2876-3 AO 6-21, 2870-1 NM1 and 2870-1 NM-2.

\section{Media}

Both types of strains were grown overnight at $37^{\circ} \mathrm{C}$ in Luria Bertani (LB) Agar, Miller (Difco, Mississauga, ON) in Petri dishes (Fischer Scientific, Ottawa, Canada) to full growth. $E$. sakazaki ${ }^{\text {lux }+g f p+}$ had $100 \mathrm{mg} / \mathrm{ml}$ ampicillin (Fischer Scientific, Ottawa, Canada) supplemented to the agar plates. Both strains were transferred to LB broth, Miller (Difco) and incubated at $37^{\circ} \mathrm{C}$, in $200 \mathrm{rpm}$ shaking incubator overnight to encourage growth.

\section{Biofilm assays}

Wild-type E. sakazakii

$200 \mu \mathrm{l}$ of overnight cultures were transferred into a polystyrene, clear bottom, Costar 96 well assay plate (Corning Incorporated, Corning, New York) and incubated overnight at $37^{\circ} \mathrm{C}$ for 1,2 and 3 day intervals. Polystyrene plates were used because E. sakazakii was found to have a strong attachment to plastic and latex ${ }^{2,10}$ and clear bottoms were necessary to measure luminescence and fluorescent in lux/gfp manipulated strains. LB broth was used as a control having no biofilm formation. In 2 and 3 day cultures, cells were removed each day and revitalized with fresh LB media. Plates were washed with $200 \mu$ l Phosphate Buffered Saline 
(PBS) (Sigma) to remove loosely associated bacteria, dried and stained with $160 \mu$ l concentrations of $0.2 \%-0.4 \%$ crystal violet (EM Science, Gibbstown, NJ) for 5 minutes. The strongest biofilm forming strain was chosen and optimized using a recursive method to determine a crystal violet concentration that would produce an absorbance values from 0.001 to 0.099 for the control. A concentration of $0.3 \%$ crystal violet was later chosen as final concentration. Wells were washed three times with $200 \mu \mathrm{l}, 220 \mu \mathrm{l}$ and $220 \mu \mathrm{l}$ respectively of PBS. After completely air drying, plates were destained by adding $160 \mu \mathrm{l}$ of $95 \%$ ethanol and incubated at room temperature for $15 \mathrm{~min}$. Optical density was measured at $\mathrm{OD}_{590}$.

\section{Bioluminescent E. sakazakii}

Cell lines were cultured in 96 well plates and cultured like the wild type for 1,2 and 3 day intervals, and revitalized with fresh media each day. Cells were removed, washed with 200 $\mu 1 \mathrm{LB}$ broth and visualized. The NightOwl Molecular Light Imager (EG \& G Berthold, Germany) detected luminescence by illuminating plates at a medium setting for 20 seconds, and a transilluminator was used to visualize fluorescence. For quantitative measurements, plates were measured for fluorescence at excitation and emission rates of 355/460nm and $485 / 535 \mathrm{~nm}$ and for luminescence in the Victor $^{2}$ Multilabe Counter (Wallac, Waltham, Mass.) for detection of biofilm forming $E$. sakazakii.

\section{Statistical Analysis}

The unpaired, one-sided $t$-test and Gaussian distribution normality tests was used in the Graph Pad Prism program (Graph Pad Software, San Diego) to obtain p-values for differences between means in assessing the significance of biofilm formation of each strain over a given time period. Pvalues $\leq 0.05$ were designated to indicate a significant effect on biofilm formation, with values $\leq 0.01$ being very significant and values $\leq 0.001$ being extremely significant. Values $\geq 0.05$ were designated insignificant. Comparisons were made between each $E$. sakazakii strain between 1 day and 2 day biofilm formation and between marked cells between 1, 2 and 3 days. Trends between the conventional and novel methods were compared to evaluate effectiveness of novel system in detecting biofilm formation.

\section{RESULTS}

\section{Optimizing crystal violet concentration}

Crystal violet concentrations of $0.05,0.075,0.1,0.2,0.3,0.4$ and 0.5 were applied to the strongest growing $E$. sakazakii strain, ES 3253, to determine what concentration would yield an absorbance value of $\leq 0.1$ for the control of LB and $\geq 0.1$ for stained biofilm. Crystal violet concentration of $0.3 \%$ was determined to yield favorable results and was used for studying biofilm formation of all eight strains of $E$. sakazakii.

\section{Biofilm formation of 8 strains after 1 and 2 days}

E. sakazakii strains were cultured and stained with $0.3 \%$ crystal violet to examine biofilm formation. There was no significant effect after 1 and 2 days in strains ES 2855 ( $\mathrm{p}=$ $0.0566)$, ES $2870(\mathrm{p}=0.4175)$ and ES $3263(\mathrm{p}=0.3640)$ (Figure 1). There was a significant increase in biofilm formation in ES $2876(\mathrm{p}=0.0204)$ and a very significant increase between day 1 and 2 in ES 2871 (0.0039) (Figure 1). There was an extremely significant increase in ES 3253 $(\mathrm{p}=0.0007)$ (Figure 1). ES $5199(\mathrm{p}=0.0146)$ and ES 51329 $(p=0.0055)$ were the only strains that had statistically significant decreases in biofilm formation on the second day.

Biofilm formation of 4 strains with reporter system measuring luminescence

Cultures of E. sakazakii ${ }^{l u x+g f p+}$ strains were measured for luminescence to determine change in biofilm formation over 1,2 and 3 days by detecting cell metabolic activity. 2876-3 A08-12 had a significant increase $(\mathrm{p}=0.0029)$ in metabolic activity in the biofilm between days 1 and 2 , and a significant decrease between days 2 and 3 (Figure 2). 2876-3 A06-21 had an extremely significant $(p=0.0002)$ decrease in metabolic activity in the biofilm between days 1 and 2 . No difference was noticed for day 3 (Figure 2). 2870-1 NM-1 and 2870-1 NM-2 both showed no significant difference in metabolic activity between days 1,2 and $3(\mathrm{p}=0.0997$ and $\mathrm{p}=0.0977$ respectively) (Figure 2).

Biofilm formation of 4 strains with reporter system measuring fluorescence

Cultures of $E$. sakazakii ${ }^{l u x+g f p+}$ strains were measured for fluorescence to determine change in biofilm formation over 1,2 and 3 days by detecting total cell biomass. No significant difference was noted in any of the strains over 1, 2 and 3 days.

\section{Comparison of conventional and novel biofilm detection methods}

Conventional methods were compared with novel methods to determine if the two methods showed similar trends in biofilm formation. ES 2876 was compared with 2876-3 AO8-12 and 2876-3 AO6-21, with ES 2876 showing a significant increase and 2876-3 AO8-12 showing a very significant increase in biofilm formation between days 1 and 2. 2876-3 AO6-21 showed a significant decrease in biofilm formation. ES 2870 was compared to 2870-1 NM-1 and 2870-1 NM-2 with all three showing no significant difference between day 1 and day 2 (Figure 3).

\section{DISCUSSION}

The present experiments were undertaken to investigate the effectiveness of the novel dual marker system in detecting and studying biofilm formation when compared to 


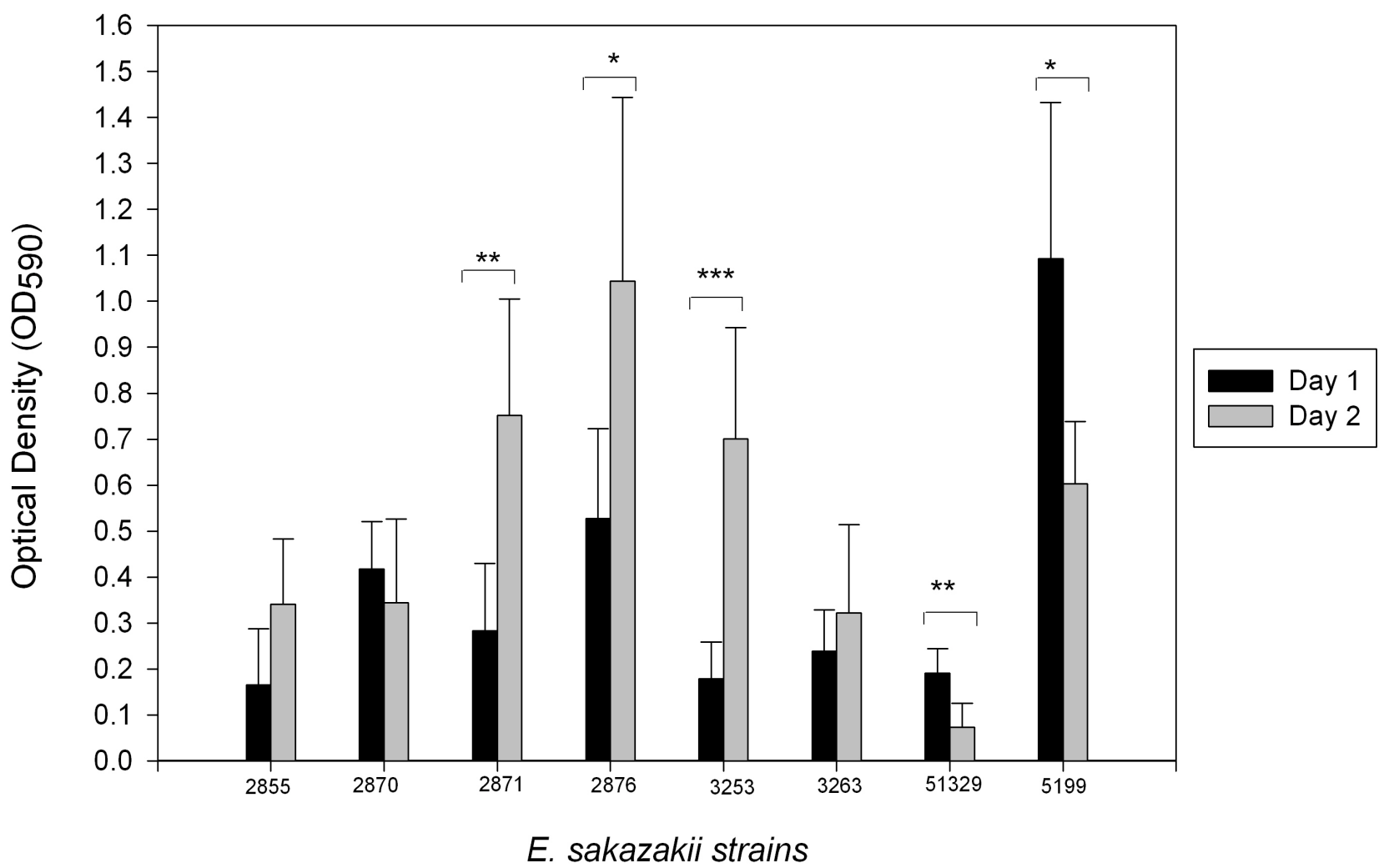

Figure 1: Average data using conventional method to determine change in biofilm formation of 8 E. sakazakii strains between 1 and 2 days. 8 strains of $E$. sakazakii were cultured to determine the change in biofilm formation over 2 days. Strains were set up in replicates of 6 and positioned in wells to minimize plate positional effects. LB broth was used as a control to be used to determine change in biofilm growth. Cells were cultured overnight at $37^{\circ} \mathrm{C}$ for 1 or $2 \mathrm{~d}$ to allow for biofilm formation, washed once with PBS to remove loosely associated cells and stained with $0.3 \%$ crystal violet. Cells were washed 3 times with PBS to remove excess stain and destained with $95 \%$ ethanol. Uptake of dye was measured by a spectrophotometer at 590nm. Data illustrated are the means and standard errors of the optical densities that are correlated to biofilm formation. Significance of difference between treatments was tested using Students $t$-test and p-values were obtained to determine statistical significance of biofilm formation in each strain when compared to the control over 2 days. Statistical significance was determined by * representing statistically significant effect on biofilm formation where $p$ $\leq \mathbf{0 . 0 5}, * *$ representing a very significant difference with $\mathbf{p} \leq \mathbf{0 . 0 1}$ and $* * *$ representing an extremely significant difference where $p$ values are $\leq \mathbf{0 . 0 0 1}$. Values $\geq 0.05$ were designated insignificant and were not marked.

conventional methods. Prior experiments found that $E$. sakazakii adheres strongly and forms a biofilm on latex and plastic $^{2,4}$ but more in-depth studies on the behaviors of biofilms and their growth limits were limited by conventional methods. Subsequent treatments to prevent or remove biofilms have failed due to the lack of understanding of E. sakazakii. To address this, a novel method for studying biofilm formation was studied here using a gene reporter system to measure biofilm formation. ${ }^{10}$

The conventional method for studying biofilm formation was optimized and used to measure the amount of biofilm formed in 8 strains of E. sakazakii over two days (Figure 1). This determined the extent of biofilm formed and the behavior of each strain independently at given time points. Each strain exhibits varying degrees of growth and biofilm formation and the peak points of development can be determined to be used as points where optimal studies can be performed for further understanding of biofilms. At these peak points, bacteria are at their most resistant and they are where varying conditions must be tested to develop new ways of penetrating and destabilizing the biofilms. Some strains showed a statistical significant increase in biofilm formation indicated that they attached to the surface and began forming a biofilm within the first two days (Figure 1). Strains that showed no significant difference indicated that they look much longer to attach to the surface and they could be more easily removed within the first two days. Strains that showed a significant decrease in biofilm after the first day suggests that those strains adhere and form biofilm rapidly within the first day but decrease on the second day due to the 


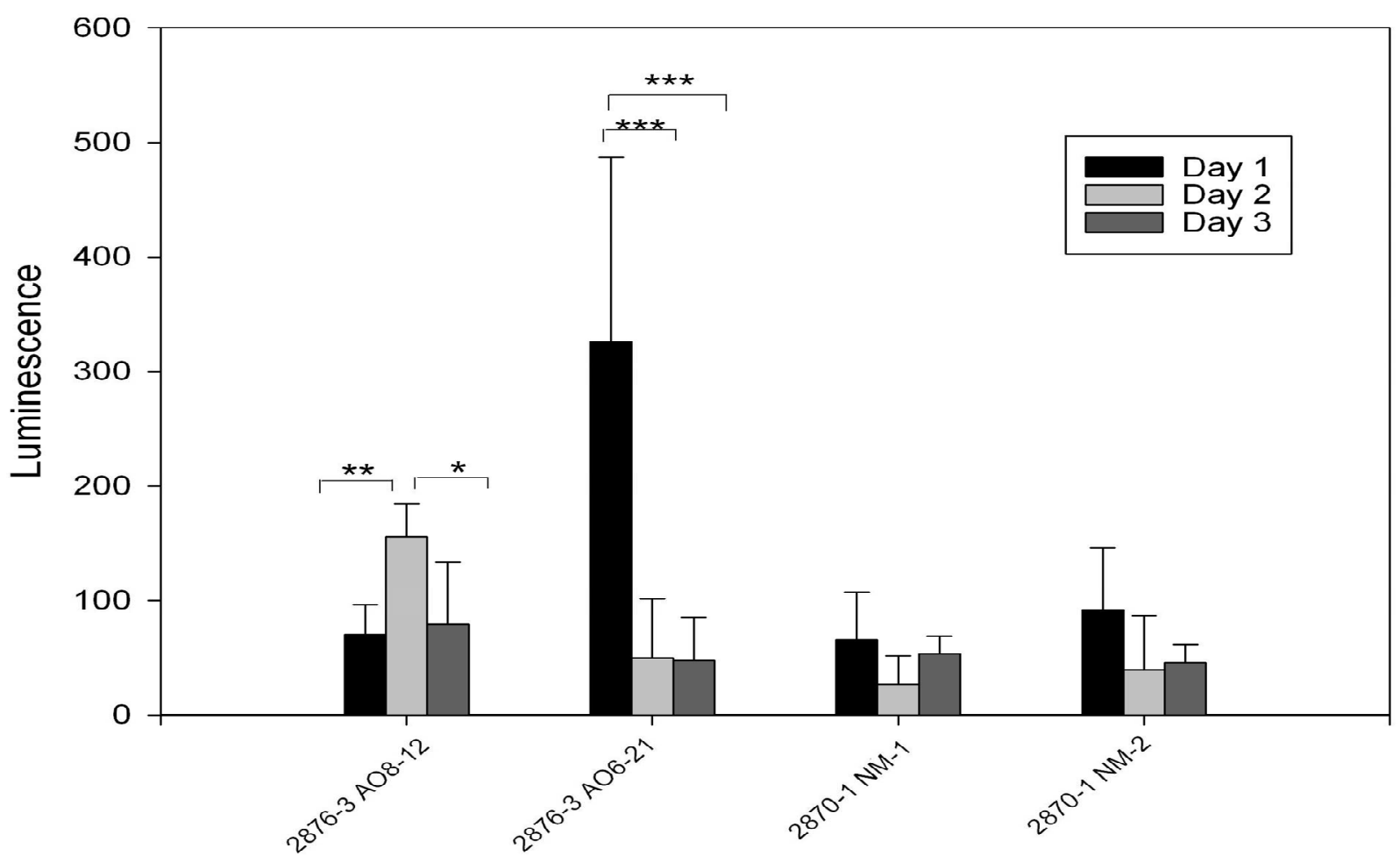

Figure 2: Average data using novel dual reporter system to determine change in biofilm formation of 4 strains of $E$. sakazakii marked with reporter system over 3 days. 4 strains of $E$. sakazakii were marked with a lux/gfp reporter system and cultured to determine change in biofilm formation over 3 days. Strains were set up in replicates of 6 and positioned to minimize plate positional effects. LB broth was used as a control to determine the change in biofilm development. Cells were cultured overnight at $37^{\circ} \mathrm{C}$ to allow for biofilm formation, washed once with $\mathrm{LB}$ to remove loosely associated cells and measured for luminescence using the Victor $^{2}$ multilable cell counter. Data illustrated are the means and standard errors of the luminescence, detected from the lux reporter, correlated to the metabolic activity of the cells in the biofilm. Significance of difference between treatments was tested using Students $t$-test and Gaussian normality tests and the pvalues were obtained to determine statistical significance of biofilm formation between each time period. For biofilm formation over day 2 and 3 , the same cells were refreshed with cell culture and allowed to incubate till the next measurements. Statistical significance was determined by * representing statistically significant effect on biofilm formation where $\mathbf{p} \leq \mathbf{0 . 0 5}, * *$ representing a very significant difference with $\mathbf{p} \leq \mathbf{0 . 0 1}$ and $* * *$ representing an extremely significant difference where $p$ values are $\leq \mathbf{0 . 0 0 1}$. Values $\geq 0.05$ were designated insignificant and were not marked.

lack of nutrients or space available (Figure 1). For further understanding of each strain, strains would need to be studied for a longer period of time. Studying the bacteria using this method, especially over a time line is challenging because once bacteria are stained with toxic crystal violet they die and cannot be analyzed further at later times or in altered conditions. Accurate behavioral studies are not acquired if cells can not be measured and returned for further growth until next measurement. Crystal violet is also a very strong staining reagent that can produce false positives adding to the ineffectiveness of conventional methods. The differences in biofilm formation of each strain are also used in determining the effectiveness of the novel system by comparing growth trends between the two methods.

We tested our dual marker system with 4 strains of $E$. sakazakii to determine if it could be used for in situ monitoring of the biomass and metabolic activity of the cells in the biofilm. The lux gene was detected by luminescence which is proportional to metabolic activity of cells and correlated to biofilm formation. This novel system is advantageous because it does not require the use of crystal violet thus eliminating the use of toxic dyes and reduces inaccuracy of results. It is also undisruptive to cells in the biofilm which allows for monitoring at longer time intervals and under varying conditions. 2876-3 AO8-12 had a statistically significant increase in biofilm development between day 1 and 2, with a statistically significant decrease at day 3 suggesting that this strain reaches its peak formation at day 2 and then decreases at day 3 (Figure 2). 2876-3 AO6-21 had its maximum biofilm formation at day 1 , and then displayed a significant decrease in days 2 and 3 indicating that its attachment and growth rate is more rapid 
i)

a)

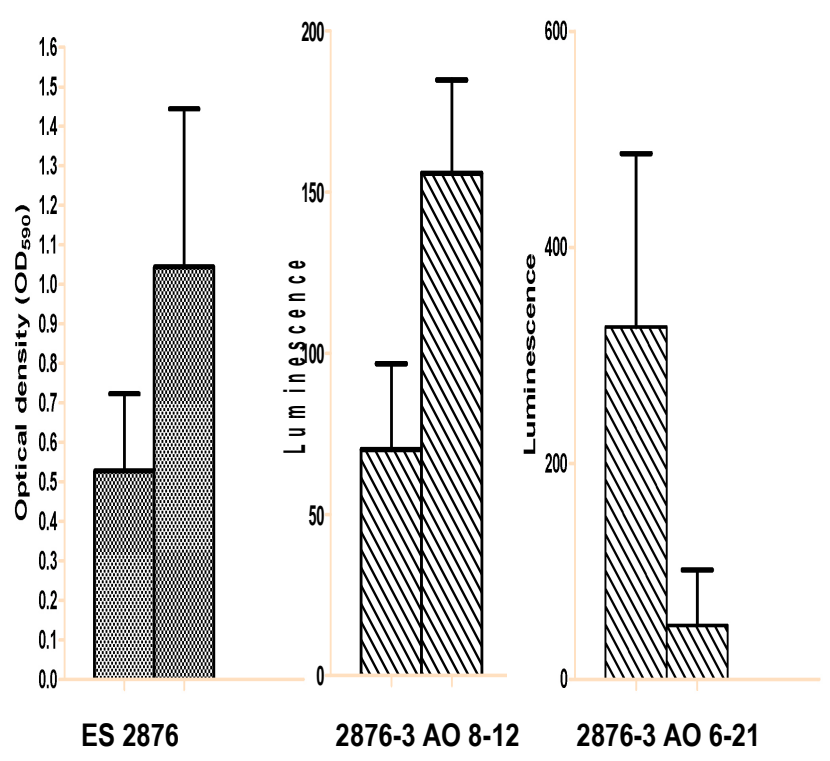

E. sakazakii wild type and marked strains ii)
a)
b)
c)

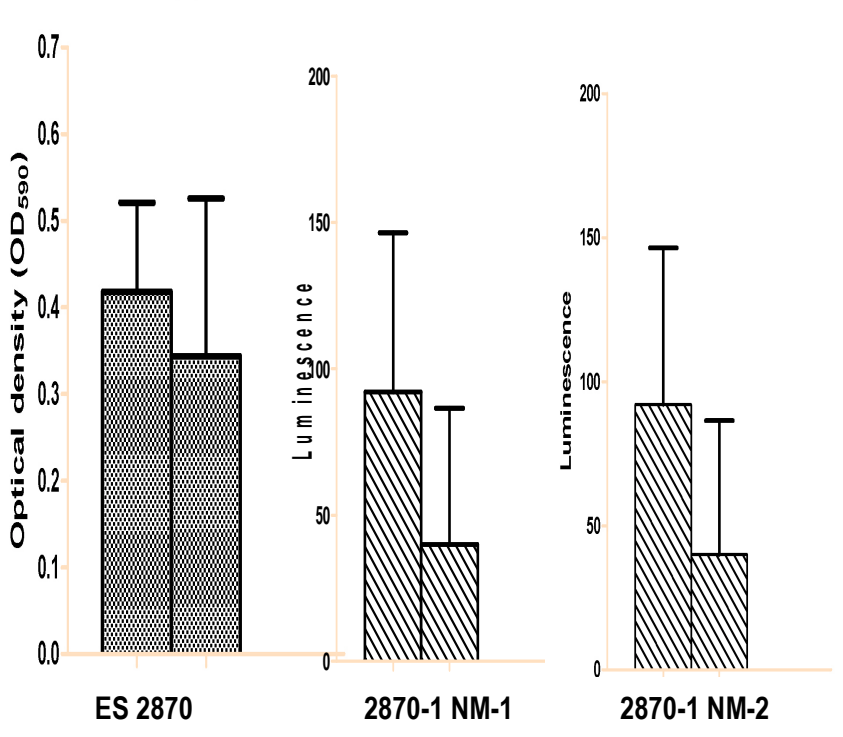

E. sakazakii wild type and marked strains

Figure 3: Comparison of conventional methods and novel dual reporter system method to determine effectiveness of novel system in detecting changes in biofilm formation. Strains of $E$. sakazakii cultured as described in Figure 1 and 2 were combined and compared for similar trends in biofilm formation between the conventional and novel methods over 2 days. i) Data illustrated here is comparison of change in biofilm formation of (a) ES 2876 using the conventional method compared to (b) 2876-3 AO 8-12 and (c) 2876-3 AO 6-21 detected using the new reporter system.

ii) Data illustrated here is comparison of change in biofilm formation of (a) ES 2870 using the conventional method compared to (b) 2870-1 NM-1 and (c) 2870-1 NM-2 using the novel system. Significance of difference between the two systems was determined based on Student $t$-test and p-values determined in Figures 1 and 2, and significant differences detected in each method were compared.

than the aforementioned strain (Figure 2). The significant decreases in these two strains can be attributed to a decrease in biofilm formation, but because the lux gene only detects metabolic activity, the decrease can be correlated to the stationary phase that cells enter once at full growth. For further investigation, the second marker used, $g f p$, must be employed. 2870-1 NM-1 and 2870-1 NM-2 showed no statistical difference in activity over the 3 day period, indicating that they attach much slower to surfaces over the first 3 days and there is little or no biofilm formed. Further analysis over a longer period of time is necessary to determine when these cells adhere to surfaces and begin producing a biofilm. The highest and lowest metabolic activity, related to the cells peak growth phase and stationary phase respectively, can be determined and used to study the bacteria at different conditions to determine when they are more resistant; in their growth phase or once in stationary phase.
The second marker in this reporter system, the $g f p$ reporter, is fluorescent and has no energy requirements, thus will fluoresce under all growth phases and will be proportional to cell biomass. Used in conjunction with the lux reported, the metabolic activity and the biomass of cells in the biofilm can be determined. This reporter is important because the lux reporter used alone can not accurately understand biofilm formation, measuring only metabolic activity and not actual biomass. The fluorescence of the protein encoded by the $g f p$ gene, according to literature can be measured at an excitation range between 395 to $398 \mathrm{~nm}$ and emission ranges between 475 to $478 \mathrm{~nm}$. Filters on the Victor $^{2}$ were not available at these wavelengths and thus the closest filters were chosen at excitation values of $355 \mathrm{~nm}$ and $485 \mathrm{~nm}$ and emission values of $460 \mathrm{~nm}$ and $535 \mathrm{~nm}$ respectively. No significant difference was detected between any of the strains of $E$. sakazakii marked with the $g f p$ reporter genes at either of these wavelengths. Biomass of cells in biofilm was not able to be 
determined, thus further inferences on whether the biofilms were varying in biomass or just metabolic activity could not be made based on just the lux reporter. Further testing must be performed using filters that are closer to the excitation and emission values of the $g f p$ reporter for further analysis of biofilms.

To determine the effectiveness of the new reporter system, it must be compared to the conventional method and evaluated on whether the results on biofilm formation of each strain are similar. Trends between biofilms formation of each strain at a given time point must be similar to establish that the novel method is not only effective but also accurate.

Figure 3(i) compares wild type strain ES 2870 used in the conventional methods to 2870-1 NM-1 and 2870-1 NM-2 used in the novel methods. When compared, ES 2870 in the conventional method did not show a significant change in biofilm formation between days 1 and 2, and the same results were observed when measured using the reporter system. ES 2876 of the conventional method was compared next to its cloned strains used in the new methods, 2876-3 AO 8-12 and 2876-3 AO 6-21 (Figure 3 (ii)). ES 2876 displayed a statistically significant increase in biofilm formation after 2 days and the same result was noted in 2876-3 AO 8-12. 2876-3 AO 6-21 showed a significant decrease in biofilm formation, and this could be attributed to this being its metabolic activity, where it has entered its stationary phase more rapidly that 2876-3 AO 8-12. Fluorescence data again is needed here to make further conclusions about the decrease in biofilm when compared to the other 2876 strains. The nutrients available, time needed for strains to attach, and change in behaviors due to insertion of genes must also be considered and studied.

Future research needed include acquiring filters that emit the correct wavelengths to detect the $g f p$ reporter so that results on cell biomass in biofilms can be determined and correlated to the metabolic activity produced by the lux reporter. The strains can then be investigated on their ability to persist and survive under various environmental conditions including $\mathrm{pH}$, temperature, osmotic pressure and desiccation.

The present results are preliminary but determine that the new methods introduced, luminescence and fluorescence reporter systems, are rapid, simple to use and effective for studying the behavior of E. sakazakii when forming biofilms. A visible trend is noticed between the two methods when comparing the two types of strains, and therefore it can be determined that the new method can be used for further analysis. Each E. sakazakii strain exhibits a different ability to form biofilms and results produced using the novel method are more accurate because the attached cells can be studied in situ non-destructively allowing for better analysis of biofilm. The novel method is faster to perform than conventional methods, does not use toxic staining and can be monitored at multiple time intervals for each strain. This method will allow researchers to rapidly screen for differences between strains and growth conditions to determine and implement improved processes in the infant milk formula manufacturing industry and neonatal care environments to inhibit attachment, deter biofilm formation and eliminate established biofilms.

\section{Acknowledgements}

This research was supported in part by the Canadian Research Institute for Food Safety (CRIFS) as well as funded by the Dairy Farmers of Ontario (DFO). Dr. Franco J. Pagotto, Health Canada, Ottawa is thanked for providing the Enterobacter sakazakii strains. I would also like to thank my advisor Dr. Mansel Griffiths for this opportunity and my supervisor Dr. Haifeng Wang for his support, teaching and guidance. Finally, I'd like to thank rest of the staff and researchers at the Canadian Research Institute for Food Safety.

\section{REFERENCES}

1. Djordjevic, D., Wiedmann, M. and McLandsborough, L.A. 2002. Appl. and Enviro. Micro. 86(6): 2950-2958

2. Iversen, C., Lane, M., Forsythe, S.J. 2004. The growth profile, thermotolerance and biofilm formation of Enterobacter sakazakii grown in infant formula milk. Letters in Appl. Micro. 38: 378-382

3. Kim, H., Ryu, J. and Beuchat, L.R. 2006. Attachment of and biofilm formation by Enterobacter sakazakii on stainless steel and enteral feeding tubes. Appl. Environ. Microbiol. 72(9): 5846-5856

4. Lee Wong, A. 1998. Biofilms in food processing environments. J. Dairy Sci. 81: 2765-2770

5. Lehner, A., Riedel, K., Eberl, L., Breeuwer, P., Diep, B. and Stephan, R. 2005. Biofilm formation, extracellular polysaccharide production and cell-to-cell signaling in various Enterobacter sakazakii strains. Aspects promoting environmental persistence. J Food Prot. 68(11): 2287-2294

6. Leon Ohl, A., Horn, H. and Hempel, D.C. 2004. Behaviour of biofilm systems under varying hydrodynamic conditions. Wat. Scie. And Technol. 49(11-12): 345-351

7. Nazarowec-White, M. and Farber, J.M. 1997. Enterobacter sakazakii: a review. Int. J. Food Microbiology. 34: 103-113

8. Van Acker, J., de Smet, F., Muyldermans, G., Bougatef, A., Naessens, A. and Lauwers, S. 2001. Outbreak of Necrotizing Enterocolitis Associated with Enterobacter sakazakii in Powdered Milk Formula. Jour. Clinical. Micro. 39(1): 293-297

9. World Health Organization (WHO). 2004. Enterobacter sakazakii and other microorganisms in powdered infant formula: Epidimiology and public health aspects. 2: 5-9.

10. Unge, A., Tombolini, R., Molbak, L. and Jansson, J.K. 1999. Appl. Environ. Microbiol. 65(2): 813-821 